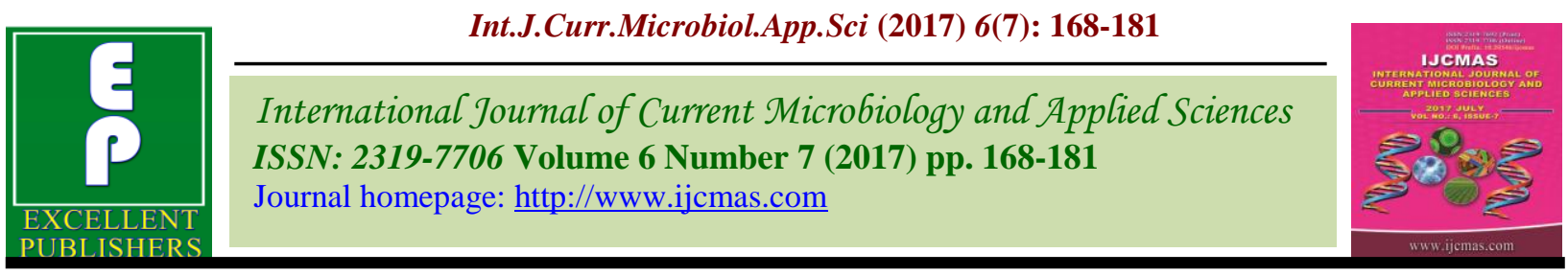

Original Research Article

https://doi.org/10.20546/ijcmas.2017.607.020

\title{
PEG Induced Screening for Drought Tolerance in Tomato Genotypes
}

\author{
P. Arun Kumar ${ }^{1 *}$, N.N. Reddy ${ }^{2}$ and N. Jyothi Lakshmi ${ }^{2}$ \\ ${ }^{1}$ Division of Crop Improvement, ICAR-CTCRI, Thiruvananthapuram, Kerala, India \\ ${ }^{2}$ Division of Crop Science, ICAR-CRIDA, Hyderabad, Telangana, India \\ *Corresponding author
}

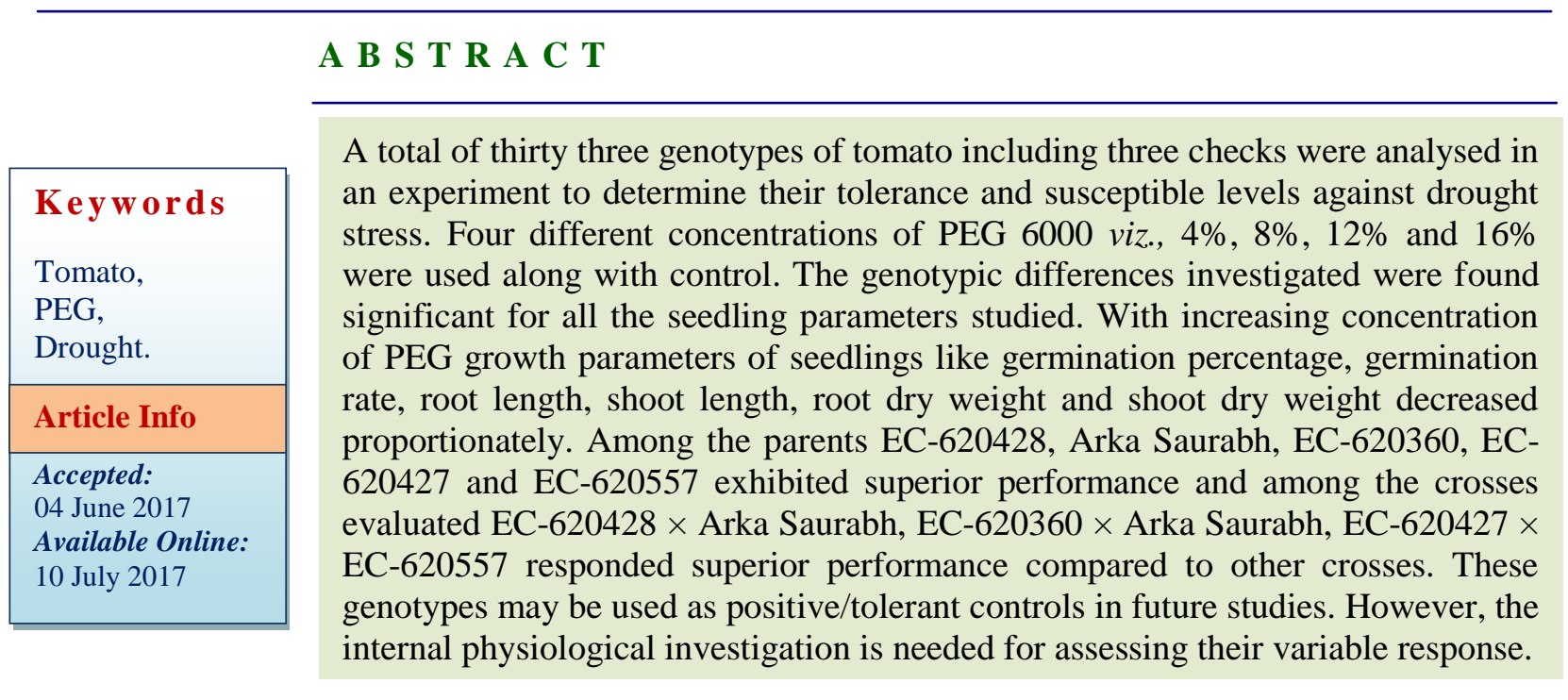

\section{Introduction}

Among the various abiotic stresses elevated temperatures and drought are the main (Pena and Hughes, 2007). Tomato is one of the widely grown vegetables in the world. It is consumed in fresh form as salad and in various processed forms like soup, sauce, ketchup, paste, puree, powder and canned whole fruit etc. and tops the list of processed vegetables. Polyethylene glycol (PEG), a series of polymers that vary from viscous liquids to waxy solids has been used to induce water stress artificially (Larher et al., 1993). PEG induced osmotic stress is found to reduce cell water potential (Govindaraj et al., 2010). An increase in concentration of PEG6000 , resulted a decrease in germination rate, root length, shoot length and seed vigour in certain crop plants (Khodarahmpour, 2011). Tomato has been selected for better growth under PEG simulated water stress (Bressan et al., 2003). In vitro selection techniques involving the use of PEG, is one of the reliable methods for screening desirable genotypes and to study further the effects of water scarcity on plant germination indices (Sakthivelu et al., 2008). Tomato genotypes tend to exhibit limited and inadequate genetic variability for drought tolerance. Hence the best way to mitigate the effects of drought stress involves the crossing of cultivated tomato with drought tolerant lines (Pena and Hughes, 2007). The present study aims to 
evaluate drought tolerant potential and compare the behaviour of different tomato genotypes under PEG simulated water stress.

\section{Materials and Methods}

The study was conducted at Horticulture Laboratory of Central Research Institute for Dry Land Agriculture (CRIDA), Hyderabad during 2016. The method suggested by Panchalingam (1983) and Babu et al., (1985) was followed to screen the genotypes against moisture stress environment under laboratory conditions. Thirty crosses of tomato along with three commercial checks viz., Arka Rakshak, US-440 and NS-516 were screened for moisture stress tolerance by employing germination test in an osmotic solution of Polyethylene Glycol (PEG-6000). Simultaneously, all the accessions were allowed to germinate in distilled water. Three replications of 10 seeds for each accession were counted and distributed over two layers of paper towel $(11 \times 11 \mathrm{~cm})$ previously moistened with water equivalent to three times the dry weight of the paper and tied both ends with rubber band and kept in a plastic tray with different concentrations $(4 \%$, $8 \%, 12 \%$ and $16 \%$ ) of Polyethylene Glycol (PEG-6000). Germination percentage was recorded for every 7 days. At the end of the $21^{\text {st }}$ day, final germination per cent, germination rate, root length, shoot length, root dry weight and shoot weight was recorded in Polyethylene Glycol (PEG-6000) as against the distilled water was calculated. The experiment was designed as a completely randomized design with two factors. The first factor was the genotypes and the second one is external water stress treatments. The sheets were rolled and placed vertically in plastic beaker, covered with polythene bag and placed at $25 \pm 1^{\circ} \mathrm{C}$ in an illuminated germinator. A seed was considered to be germinated when the emerging radicle elongated to $1 \mathrm{~mm}$. Radicle length, hypocotyl length, seedling fresh weight and dry weight were calculated as described by Uniyal et al., (1998). Observations were recorded on germination percentage, germination rate, root length $(\mathrm{cm})$, shoot length $(\mathrm{cm})$, root dry weight (mg) and shoot dry weight (mg). Analysis of variance was carried out as described by Steel et al., (1997). Statistical significance of means was tested by SPSS package.

\section{Results and Discussion}

Significant differences were observed under different PEG-6000 concentrations of $0,4 \%$, $8 \%, 12 \%$ and $16 \%$. The analysis of variance showed significant differences among the genotypes and treatments (Table 1).

\section{Germination percentage (\%)}

A decline in seed germination percentage under increasing stress has been observed (Table 2). Significant differences were observed for germination percentage between the genotypes and different PEG-6000 concentrations. Irrespective of the PEG-6000 concentrations, EC-620407 × EC-620557 recorded significantly maximum germination percentage $(80.57 \%)$ compared to all other genotypes. The germination percentage was lowest $(38.91 \%)$ in the cross EC-619982 $\times$ EC-620557. The interaction effect between the genotype and PEG-6000 concentrations was found non-significant. In $4 \%$ concentration of PEG-6000, the cross EC$620428 \times$ Arka Saurabh exhibited maximum (91.66) and EC-619982 $\times$ Arka Saurabh exhibited minimum (41.59) germination per percentages. While at $8 \%$ concentration of PEG-6000, genotype EC-620428 $\times$ Arka Saurabh $(91.06 \%)$ and EC-619982 $\times$ Pusa Ruby (34.59\%) exhibited maximum and minimum germination percentage respectively. In case of $12 \%$ concentration of PEG-6000, genotypes EC-620360 × Arka 
Saurabh $(77.70 \%)$, EC-620360 × Pusa Ruby $(26.20 \%)$ had responded as maximum and minimum germination percentage respectively. With $16 \%$ concentration of PEG-6000, EC-620407 $\times$ EC-620557 (73.21\%) responded maximum and minimum in EC-620360 × Pusa Ruby (20.52\%).

Maximum seed germination percentage was observed under control condition. It was observed that germination percentage with decreasing water potential of the environment probably was triggered by the low hydraulic conductivity of the environment where, PEG 6000 makes water unavailable to seeds, affecting the imbibition process of the seed which is fundamental for germination. In this study drought stress caused the germination percentage decrease in all of the genotypes. PEG causes the seed reserves materials hydrolysis decrease and finally the germination percentage decrease (Bhatt and Srinivasa 1987, Munns and Weir, 1981, Kulkarni and Desphpande, 2007, Aazami et al., 2010).

The germination rapidity of tolerant genotypes to the drought stress was more than the sensitive genotypes. Results of the current study were in agreement with other experiments in different plants including Ravi et al., (2011) and Alejandra et al., (2010).

\section{Germination rate}

Significant differences were observed for germination rate between the genotypes and different PEG-6000 concentrations (Table 3). Irrespective of the PEG-6000 concentrations, EC-620360 $\times$ Arka Saurabh recorded significantly maximum germination rate (7.47) compared to all other genotypes.

The germination rate was lowest (3.57) in the genotype EC-619982 × Arka Saurabh. The interaction effect between the genotype and
PEG-6000 concentrations was found significant. In $4 \%$ concentration of PEG6000, maximum and minimum germination rate was recorded in EC-620428 $\times$ Arka Saurabh (9.14) and EC-619982 $\times$ Arka Saurabh (3.53) genotype respectively. While at $8 \%$ concentration of PEG-6000, genotype EC-620428 $\times$ Arka Saurabh (8.17) and Arka Rakshak (3.28) exhibited maximum and minimum germination rate respectively. In case of $12 \%$ concentration of PEG-6000, maximum and minimum germination rate recorded in EC- $620360 \times$ Arka Saurabh (7.48) and EC-620360 × Pusa Ruby (2.27) while with $16 \%$ maximum and minimum germination rate recorded in EC-620360 $\times$ Arka Saurabh (5.93) and EC-620360 × Pusa Ruby (1.72) are presented in Fig. 9.

Results of the current study were in agreement with other experiments in different plants including Kalefetoglu et al., (2009) in chickpea. The rate of germination seems to be more sensitive to the water stress than final germination percentage caused a significant increase in mean germination time and decrease in germination. Present studies are intensely supported by the finding of Uniyal et al., (1998).

The germination responses show that tolerant genotype have high germination capacity under drought stress (Zhu et al., 2006 and Abdel-Raheem et al., 2007).

\section{Root length (cm)}

Root plays a major role in plant survival during drought and also drought tolerant can be characterized by extensive root growth (Table 4). Significant differences were observed for root length between the genotypes and different PEG-6000 concentrations. Irrespective of the PEG-6000 concentrations, EC-620428 × Arka Saurabh recorded significantly maximum root length 
$(6.74 \mathrm{~cm})$ compared to all other genotypes. The root length was lowest $(2.93 \mathrm{~cm})$ in the genotype EC-619982 $\times$ EC-620557. The interaction effect between the genotype and PEG-6000 concentrations was found significant.

In $4 \%$ concentration of PEG-6000, maximum and minimum root length was recorded in EC-620407 $\times$ Arka Abha $(8.02 \mathrm{~cm})$ and EC$619982 \times$ EC- EC-620557 $(3.38 \mathrm{~cm})$ genotype respectively. While at $8 \%$ concentration of PEG-6000, genotype EC-620428 × Arka Saurabh $(7.47 \mathrm{~cm})$ and EC-619982 $\times$ EC$620557(3.12 \mathrm{~cm})$ exhibited maximum and minimum root length respectively.

In case of $12 \%$ concentration of PEG-6000, genotypes EC-620407 × EC-620557 (6.05 $\mathrm{cm})$ and EC-620428 $\times$ Pusa Ruby $(2.34 \mathrm{~cm})$ had responded as maximum and minimum root length respectively. In case of $16 \%$ concentration of PEG-6000, genotypes EC$620407 \times$ Arka Abha $(5.14 \mathrm{~cm})$ and EC$6619982 \times$ Arka Abha $(1.43 \mathrm{~cm})$ had responded as maximum and minimum root length respectively.
Root length is an important trait against drought stress in plant varieties, with longer root growth has resistant ability for drought (Leishman and Westoby, 1994). Early and rapid elongation of roots is an important indication of drought tolerance. Ability of continued elongation of root under water stress and longer root length at deeper layer are useful in extracting water in upland conditions (Kim et al., 2001, Narayan, 1991). The plant embryo grows at germination and progresses radicles that become the primary roots and penetrate down into the soil. After radicle emergence, hypocotyl emerges and lifts the growing tip above the ground. Under drought stress condition, the root develops faster than the hypocotyls to acclimatize the drought stress.

Therefore, the growth of radicle and hypocotyls should reflect the adaptability of plant to drought stress (Zhu et al., 2006). Similar results were observed by Kulkarni and Deshpande (2007). Ability of continuous elongation of root under situation of water stress was a remarkable character of some genotypes.

Table.1 Analysis of variance for six characters in tomato

\begin{tabular}{|c|c|c|c|c|c|c|c|}
\hline \multicolumn{7}{|c|}{ Mean sum of squares } \\
\hline $\begin{array}{c}\text { Source of } \\
\text { variation }\end{array}$ & df & $\begin{array}{c}\text { Germination } \\
\%\end{array}$ & $\begin{array}{c}\text { Germination } \\
\text { rate }\end{array}$ & $\begin{array}{c}\text { Root } \\
\text { length }\end{array}$ & $\begin{array}{c}\text { Shoot } \\
\text { length }\end{array}$ & $\begin{array}{c}\text { Shoot } \\
\text { dry } \\
\text { weight }\end{array}$ & $\begin{array}{c}\text { Root dry } \\
\text { weight }\end{array}$ \\
\hline $\begin{array}{c}\text { Treatment } \\
(\mathrm{A})\end{array}$ & 4 & $383.79 * *$ & $1.01^{* *}$ & $1.13^{* *}$ & 15.61 & $967^{* *}$ & $6.72^{* *}$ \\
\hline $\begin{array}{c}\text { Genotype } \\
(\mathrm{B})\end{array}$ & 32 & $2225.64^{* *}$ & $76.43^{* *}$ & $16.75^{* *}$ & $15.66^{* *}$ & $942^{* *}$ & $47.73^{* *}$ \\
\hline $\mathrm{A} \times \mathrm{B}$ & 156 & 596.67 & 6.50 & 6.02 & 18.99 & 1517.05 & 12.99 \\
\hline Error & 424 & 10.38 & 0.08 & 0.06 & 0.07 & 3.84 & 0.08 \\
\hline
\end{tabular}


Table.2 Effect of different concentration of PEG-6000 (control, 4\%, 8\%, $12 \%$ and $16 \%$ on germination percentage of tomato genotypes

\begin{tabular}{|c|c|c|c|c|c|c|}
\hline \multirow{3}{*}{ Crosses/Hybrids } & \multicolumn{6}{|c|}{ Germination percent $(\%)$} \\
\hline & \multicolumn{6}{|c|}{ Treatment } \\
\hline & Control & $4 \%$ & $8 \%$ & $12 \%$ & $16 \%$ & Mean \\
\hline EC-620407 × Arka Saurabh & 73.54 & 69.29 & 67.01 & 59.95 & 42.56 & 62.47 \\
\hline EC-620407 × Arka Abha & 79.76 & 79.25 & 60.45 & 48.94 & 47.57 & 63.19 \\
\hline EC-620407 × Punjab Chhuhara & 84.37 & 75.99 & 70.59 & 63.63 & 43.79 & 67.67 \\
\hline EC-620407 × Pusa Ruby & 65.21 & 63.58 & 62.49 & 55.55 & 54.50 & 60.27 \\
\hline EC-620407 × EC-520078 & 85.66 & 72.38 & 72.31 & 60.55 & 47.49 & 67.68 \\
\hline EC-620407 × EC-620557 & 86.39 & 83.91 & 82.46 & 76.89 & 73.21 & 80.57 \\
\hline EC-620428 × Arka Saurabh & 93.16 & 91.66 & 91.06 & 65.92 & 52.45 & 78.85 \\
\hline EC-620428 $\times$ Arka Abha & 73.77 & 69.62 & 54.78 & 43.09 & 36.83 & 55.62 \\
\hline EC-620428 × Punjab Chhuhara & 69.33 & 50.81 & 34.62 & 30.32 & 28.56 & 42.73 \\
\hline EC-620428 × Pusa Ruby & 62.79 & 56.44 & 50.64 & 50.51 & 49.45 & 53.96 \\
\hline EC-620428 × EC-520078 & 70.55 & 64.01 & 62.64 & 50.45 & 49.63 & 59.46 \\
\hline EC-620428 × EC-620557 & 68.56 & 47.55 & 46.51 & 44.04 & 39.61 & 49.25 \\
\hline EC-620360 × Arka Saurabh & 88.38 & 86.71 & 78.37 & 77.70 & 62.98 & 78.83 \\
\hline EC-620360 × Arka Abha & 88.77 & 56.61 & 54.71 & 48.10 & 33.34 & 56.31 \\
\hline EC-620360 × Punjab Chhuhara & 67.78 & 62.20 & 46.31 & 41.59 & 31.99 & 49.97 \\
\hline EC-620360 × Pusa Ruby & 69.88 & 64.30 & 45.50 & 26.20 & 20.52 & 45.28 \\
\hline EC-620360 × EC-520078 & 52.40 & 48.24 & 47.18 & 41.84 & 41.35 & 46.20 \\
\hline EC-620360 × EC-620557 & 67.47 & 64.35 & 54.05 & 38.75 & 36.58 & 52.24 \\
\hline EC-608415 × Arka Saurabh & 74.98 & 72.81 & 62.65 & 44.47 & 31.67 & 57.31 \\
\hline EC-608415 $\times$ Arka Abha & 89.26 & 87.55 & 86.17 & 59.80 & 42.06 & 72.97 \\
\hline EC-608415 × Punjab Chhuhara & 53.90 & 51.10 & 41.82 & 41.28 & 33.34 & 44.29 \\
\hline EC-608415 × Pusa Ruby & 63.86 & 42.03 & 37.23 & 35.17 & 27.90 & 41.24 \\
\hline EC-608415 × EC-520078 & 64.88 & 44.48 & 43.58 & 41.18 & 32.39 & 45.30 \\
\hline EC-608415 × EC-620557 & 61.96 & 55.10 & 53.35 & 43.15 & 40.32 & 50.77 \\
\hline EC-619982 × Arka Saurabh & 58.72 & 41.59 & 40.76 & 32.77 & 28.78 & 40.53 \\
\hline EC-619982 × Arka Abha & 82.87 & 74.21 & 64.84 & 61.64 & 54.95 & 67.70 \\
\hline EC-619982 × Punjab Chhuhara & 86.18 & 81.11 & 49.23 & 40.44 & 38.25 & 59.04 \\
\hline EC-619982 × Pusa Ruby & 55.65 & 52.25 & 34.59 & 34.35 & 33.23 & 42.01 \\
\hline EC-619982 × EC-520078 & 63.35 & 61.76 & 42.38 & 39.15 & 25.48 & 46.42 \\
\hline EC-619982 × EC-620557 & 43.58 & 43.34 & 39.68 & 35.42 & 32.52 & 38.91 \\
\hline US-440 & 55.80 & 53.56 & 49.01 & 32.57 & 22.95 & 42.78 \\
\hline Arka Rakshak & 63.66 & 58.28 & 54.58 & 51.87 & 37.92 & 53.26 \\
\hline NS-516 & 84.53 & 84.34 & 76.66 & 73.76 & 44.89 & 72.84 \\
\hline \multirow[t]{2}{*}{ Mean } & 71.24 & 63.95 & 56.30 & 48.21 & 39.97 & 55.93 \\
\hline & \multicolumn{3}{|c|}{ S.Em \pm} & \multicolumn{3}{|c|}{ CD $(0.01)$} \\
\hline Treatment (A) & \multicolumn{3}{|c|}{0.32} & \multicolumn{3}{|c|}{0.90} \\
\hline Genotype (B) & \multicolumn{3}{|c|}{0.832} & \multicolumn{3}{|c|}{2.31} \\
\hline $\mathbf{A} \times \mathbf{B}$ & \multicolumn{3}{|c|}{1.86} & \multicolumn{3}{|c|}{5.17} \\
\hline
\end{tabular}


Table.3 Effect of different concentration of PEG-6000 (control, 4\%, 8\%, 12\% and $16 \%$ on germination rate of tomato genotypes

\begin{tabular}{|c|c|c|c|c|c|c|}
\hline \multirow{3}{*}{ Crosses/Hybrids } & \multicolumn{6}{|c|}{ Germination rate $(\%)$} \\
\hline & \multicolumn{6}{|c|}{ Treatment } \\
\hline & Control & $4 \%$ & $8 \%$ & $12 \%$ & $16 \%$ & Mean \\
\hline EC-620407 × Arka Saurabh & 7.14 & 6.22 & 5.47 & 5.44 & 3.48 & 5.55 \\
\hline EC-620407 × Arka Abha & 6.58 & 6.47 & 4.63 & 4.03 & 3.11 & 4.96 \\
\hline EC-620407 × Punjab Chhuhara & 6.29 & 5.24 & 4.52 & 4.33 & 2.62 & 4.60 \\
\hline EC-620407 × Pusa Ruby & 6.46 & 6.13 & 4.80 & 4.61 & 4.57 & 5.31 \\
\hline EC-620407 × EC-520078 & 8.25 & 8.23 & 5.61 & 4.45 & 4.34 & 6.17 \\
\hline EC-620407 × EC-620557 & 6.71 & 5.43 & 5.07 & 5.02 & 4.40 & 5.33 \\
\hline EC-620428 × Arka Saurabh & 9.37 & 9.14 & 8.17 & 5.72 & 4.77 & 7.43 \\
\hline EC-620428 × Arka Abha & 6.62 & 6.10 & 5.05 & 3.70 & 3.29 & 4.95 \\
\hline EC-620428 × Punjab Chhuhara & 6.14 & 4.70 & 4.33 & 2.95 & 2.53 & 4.13 \\
\hline EC-620428 × Pusa Ruby & 5.87 & 5.28 & 4.64 & 4.53 & 4.43 & 4.95 \\
\hline EC-620428 × EC-520078 & 6.24 & 6.00 & 5.58 & 4.41 & 4.35 & 5.32 \\
\hline EC-620428 × EC-620557 & 7.46 & 6.48 & 4.59 & 3.99 & 3.61 & 5.22 \\
\hline EC-620360 × Arka Saurabh & 8.86 & 7.57 & 7.50 & 7.48 & 5.93 & 7.47 \\
\hline EC-620360 × Arka Abha & 8.91 & 5.01 & 4.81 & 4.61 & 3.01 & 5.27 \\
\hline EC-620360 × Punjab Chhuhara & 6.65 & 5.96 & 4.51 & 3.69 & 5.96 & 4.73 \\
\hline EC-620360 × Pusa Ruby & 6.63 & 6.51 & 4.37 & 2.27 & 1.72 & 4.30 \\
\hline EC-620360 × EC-520078 & 4.86 & 4.57 & 4.47 & 3.92 & 3.84 & 4.33 \\
\hline EC-620360 × EC-620557 & 6.61 & 6.18 & 5.23 & 3.69 & 3.45 & 5.03 \\
\hline EC-608415 × Arka Saurabh & 7.58 & 7.23 & 6.02 & 4.20 & 2.94 & 5.59 \\
\hline EC-608415 × Arka Abha & 8.73 & 8.51 & 7.16 & 5.78 & 3.74 & 6.78 \\
\hline EC-608415 × Punjab Chhuhara & 5.68 & 4.96 & 4.89 & 3.94 & 3.18 & 4.53 \\
\hline EC-608415 × Pusa Ruby & 6.25 & 4.16 & 3.42 & 3.41 & 2.56 & 3.96 \\
\hline EC-608415 × EC-520078 & 6.31 & 4.14 & 4.03 & 3.74 & 2.95 & 4.23 \\
\hline EC-608415 × EC-620557 & 5.91 & 5.32 & 5.15 & 4.26 & 3.93 & 4.91 \\
\hline EC-619982 × Arka Saurabh & 5.46 & 3.53 & 3.52 & 2.95 & 2.39 & 3.57 \\
\hline EC-619982 × Arka Abha & 8.02 & 7.22 & 6.36 & 5.88 & 5.28 & 6.55 \\
\hline EC-619982 × Punjab Chhuhara & 7.71 & 7.11 & 4.42 & 3.73 & 1.81 & 4.96 \\
\hline EC-619982 × Pusa Ruby & 4.89 & 4.81 & 3.73 & 3.04 & 2.83 & 3.86 \\
\hline EC-619982 × EC-520078 & 5.81 & 3.74 & 3.70 & 3.14 & 2.38 & 3.75 \\
\hline EC-619982 × EC-620557 & 5.85 & 3.95 & 3.78 & 3.05 & 2.54 & 3.84 \\
\hline US-440 & 5.77 & 5.12 & 4.94 & 3.81 & 3.57 & 4.64 \\
\hline Arka Rakshak & 5.52 & 5.29 & 3.28 & 2.71 & 2.18 & 3.80 \\
\hline NS-516 & 7.65 & 7.50 & 7.29 & 6.45 & 5.37 & 6.85 \\
\hline \multirow[t]{2}{*}{ Mean } & 6.75 & 5.87 & 5.00 & 4.21 & 3.54 & 5.07 \\
\hline & \multicolumn{3}{|c|}{ S.Em \pm} & \multicolumn{3}{|c|}{ CD (0.01) } \\
\hline Treatment (A) & \multicolumn{3}{|c|}{0.028} & \multicolumn{3}{|c|}{0.077} \\
\hline Genotype (B) & \multicolumn{3}{|c|}{0.071} & \multicolumn{3}{|c|}{0.198} \\
\hline $\mathbf{A} \times \mathbf{B}$ & \multicolumn{3}{|c|}{0.159} & \multicolumn{3}{|c|}{0.442} \\
\hline
\end{tabular}


Table.4 Effect of different concentration of PEG-6000 (control, 4\%, 8\%, $12 \%$ and $16 \%$ on root length of tomato genotypes

\begin{tabular}{|c|c|c|c|c|c|c|}
\hline \multirow{3}{*}{ Crosses/Hybrids } & \multicolumn{6}{|c|}{ Root length (cm) } \\
\hline & \multicolumn{6}{|c|}{ Treatment } \\
\hline & Control & $4 \%$ & $8 \%$ & $12 \%$ & $16 \%$ & Mean \\
\hline EC-620407 × Arka Saurabh & 6.40 & 6.25 & 5.31 & 5.13 & 4.49 & 5.51 \\
\hline EC-620407 × Arka Abha & 8.39 & 8.02 & 6.32 & 5.29 & 5.14 & 6.63 \\
\hline EC-620407 × Punjab Chhuhara & 6.25 & 5.13 & 4.21 & 3.11 & 3.09 & 4.36 \\
\hline EC-620407 × Pusa Ruby & 5.40 & 5.23 & 5.22 & 4.40 & 3.58 & 4.77 \\
\hline EC-620407 × EC-520078 & 7.40 & 7.40 & 6.41 & 5.12 & 5.06 & 6.28 \\
\hline EC-620407 × EC-620557 & 8.29 & 6.44 & 6.38 & 6.05 & 2.88 & 6.01 \\
\hline EC-620428 × Arka Saurabh & 8.26 & 7.91 & 7.47 & 5.68 & 4.41 & 6.74 \\
\hline EC-620428 × Arka Abha & 5.59 & 5.43 & 5.11 & 4.20 & 4.06 & 4.88 \\
\hline EC-620428 × Punjab Chhuhara & 8.35 & 7.34 & 5.79 & 5.45 & 4.23 & 6.23 \\
\hline EC-620428 × Pusa Ruby & 4.52 & 4.45 & 3.15 & 2.34 & 2.24 & 3.34 \\
\hline EC-620428 × EC-520078 & 4.78 & 4.52 & 4.51 & 4.43 & 4.36 & 4.52 \\
\hline EC-620428 × EC-620557 & 6.81 & 6.75 & 5.77 & 5.57 & 5.05 & 5.99 \\
\hline EC-620360 × Arka Saurabh & 7.63 & 7.43 & 7.41 & 5.73 & 2.29 & 6.10 \\
\hline EC-620360 × Arka Abha & 8.04 & 5.11 & 4.52 & 3.92 & 3.61 & 5.04 \\
\hline EC-620360 × Punjab Chhuhara & 7.02 & 5.86 & 5.35 & 4.21 & 4.15 & 5.31 \\
\hline EC-620360 × Pusa Ruby & 7.46 & 5.22 & 3.49 & 3.42 & 2.14 & 4.35 \\
\hline EC-620360 × EC-520078 & 4.36 & 4.15 & 3.57 & 2.43 & 2.18 & 3.34 \\
\hline EC-620360 × EC-620557 & 6.40 & 5.21 & 4.21 & 4.14 & 4.07 & 4.80 \\
\hline EC-608415 × Arka Saurabh & 7.17 & 6.47 & 5.39 & 5.28 & 2.04 & 5.27 \\
\hline EC-608415 × Arka Abha & 7.59 & 7.41 & 7.37 & 5.33 & 4.84 & 6.51 \\
\hline EC-608415 × Punjab Chhuhara & 4.89 & 3.93 & 3.58 & 3.40 & 3.13 & 3.79 \\
\hline EC-608415 × Pusa Ruby & 7.16 & 6.39 & 4.94 & 3.74 & 2.86 & 5.01 \\
\hline EC-608415 × EC-520078 & 4.76 & 3.59 & 3.58 & 2.92 & 2.19 & 3.41 \\
\hline EC-608415 × EC-620557 & 3.75 & 3.71 & 3.68 & 3.50 & 3.21 & 3.57 \\
\hline EC-619982 × Arka Saurabh & 6.00 & 5.86 & 5.75 & 4.87 & 4.67 & 5.43 \\
\hline EC-619982 × Arka Abha & 6.75 & 6.62 & 4.85 & 4.75 & 1.43 & 4.88 \\
\hline EC-619982 × Punjab Chhuhara & 7.21 & 6.75 & 4.36 & 3.71 & 2.85 & 4.97 \\
\hline EC-619982 × Pusa Ruby & 4.68 & 4.62 & 3.38 & 3.14 & 2.66 & 3.69 \\
\hline EC-619982 × EC-520078 & 6.88 & 6.04 & 4.13 & 2.81 & 1.56 & 4.28 \\
\hline EC-619982 × EC-620557 & 3.47 & 3.38 & 3.12 & 2.71 & 1.98 & 2.93 \\
\hline US-440 & 5.43 & 3.42 & 3.34 & 3.29 & 2.81 & 3.66 \\
\hline Arka Rakshak & 5.85 & 5.64 & 4.68 & 4.24 & 4.15 & 4.91 \\
\hline NS-516 & 6.18 & 6.02 & 4.95 & 4.59 & 1.27 & 4.60 \\
\hline \multirow[t]{2}{*}{ Mean } & 6.33 & 5.68 & 4.88 & 4.20 & 3.29 & 4.88 \\
\hline & \multicolumn{3}{|c|}{ S.Em \pm} & \multicolumn{3}{|c|}{ CD $(0.01)$} \\
\hline Treatment (A) & \multicolumn{3}{|c|}{0.026} & \multicolumn{3}{|c|}{0.071} \\
\hline Genotype (B) & \multicolumn{3}{|c|}{0.066} & \multicolumn{3}{|c|}{0.183} \\
\hline $\mathbf{A} \times \mathbf{B}$ & \multicolumn{3}{|c|}{0.147} & \multicolumn{3}{|c|}{0.408} \\
\hline
\end{tabular}


Table.5 Effect of different concentration of PEG-6000 (control, 4\%, 8\%, 12\% and $16 \%$ on shoot length $(\mathrm{cm})$ of tomato genotypes

\begin{tabular}{|c|c|c|c|c|c|c|}
\hline \multirow{3}{*}{ Crosses/Hybrids } & \multicolumn{6}{|c|}{ Shoot length $(\mathrm{cm})$} \\
\hline & \multicolumn{6}{|c|}{ Treatment } \\
\hline & Control & $4 \%$ & $8 \%$ & $12 \%$ & $16 \%$ & Mean \\
\hline EC-620407 × Arka Saurabh & 8.42 & 5.52 & 5.39 & 4.42 & 4.14 & 5.57 \\
\hline EC-620407 × Arka Abha & 7.41 & 5.26 & 5.24 & 4.13 & 2.01 & 4.81 \\
\hline EC-620407 × Punjab Chhuhara & 9.66 & 6.95 & 6.22 & 2.15 & 1.87 & 5.37 \\
\hline EC-620407 × Pusa Ruby & 7.55 & 6.52 & 5.05 & 4.49 & 4.28 & 5.58 \\
\hline EC-620407 × EC-520078 & 9.09 & 7.59 & 5.44 & 2.38 & 2.21 & 5.34 \\
\hline EC-620407 × EC-620557 & 8.39 & 5.17 & 4.21 & 3.96 & 3.26 & 5.00 \\
\hline EC-620428 × Arka Saurabh & 10.36 & 9.49 & 5.42 & 4.35 & 3.49 & 6.62 \\
\hline EC-620428 × Arka Abha & 7.67 & 6.77 & 4.69 & 4.62 & 1.47 & 5.04 \\
\hline EC-620428 × Punjab Chhuhara & 6.17 & 5.47 & 4.46 & 3.56 & 1.59 & 4.25 \\
\hline EC-620428 × Pusa Ruby & 6.46 & 4.15 & 4.15 & 3.63 & 1.49 & 3.98 \\
\hline EC-620428 × EC-520078 & 6.99 & 6.98 & 5.87 & 5.35 & 1.61 & 5.36 \\
\hline EC-620428 × EC-620557 & 8.38 & 7.82 & 4.14 & 3.50 & 2.11 & 5.19 \\
\hline EC-620360 × Arka Saurabh & 9.91 & 9.69 & 5.53 & 5.28 & 3.85 & 6.85 \\
\hline EC-620360 × Arka Abha & 10.80 & 7.46 & 4.54 & 3.83 & 3.05 & 5.93 \\
\hline EC-620360 × Punjab Chhuhara & 6.36 & 4.35 & 4.27 & 3.22 & 1.03 & 3.85 \\
\hline EC-620360 × Pusa Ruby & 8.60 & 6.12 & 5.32 & 1.16 & 1.03 & 4.44 \\
\hline EC-620360 × EC-520078 & 6.61 & 5.46 & 3.82 & 3.44 & 3.28 & 4.52 \\
\hline EC-620360 × EC-620557 & 8.13 & 6.54 & 4.67 & 1.38 & 1.20 & 4.38 \\
\hline EC-608415 × Arka Saurabh & 7.53 & 4.42 & 3.03 & 2.90 & 2.29 & 4.03 \\
\hline EC-608415 × Arka Abha & 9.14 & 8.35 & 8.18 & 4.27 & 3.32 & 6.65 \\
\hline EC-608415 × Punjab Chhuhara & 6.82 & 5.64 & 3.82 & 3.67 & 2.55 & 4.50 \\
\hline EC-608415 × Pusa Ruby & 8.33 & 3.53 & 2.60 & 0.70 & 0.61 & 3.15 \\
\hline EC-608415 × EC-520078 & 5.42 & 4.82 & 3.54 & 2.68 & 0.58 & 3.41 \\
\hline EC-608415 × EC-620557 & 6.17 & 4.87 & 4.23 & 3.07 & 0.72 & 3.81 \\
\hline EC-619982 × Arka Saurabh & 7.50 & 6.71 & 6.13 & 1.70 & 1.04 & 4.62 \\
\hline EC-619982 $\times$ Arka Abha & 8.21 & 3.78 & 3.75 & 2.50 & 2.47 & 4.14 \\
\hline EC-619982 × Punjab Chhuhara & 8.83 & 8.05 & 3.37 & 3.31 & 2.45 & 5.20 \\
\hline EC-619982 × Pusa Ruby & 6.68 & 5.49 & 3.48 & 2.53 & 0.45 & 3.73 \\
\hline EC-619982 × EC-520078 & 7.74 & 5.14 & 4.50 & 3.63 & 0.42 & 4.28 \\
\hline EC-619982 × EC-620557 & 4.72 & 3.11 & 2.85 & 2.33 & 0.27 & 2.66 \\
\hline US-440 & 7.34 & 5.66 & 5.46 & 4.09 & 0.43 & 4.59 \\
\hline Arka Rakshak & 6.71 & 6.45 & 2.40 & 1.58 & 0.74 & 3.57 \\
\hline NS-516 & 9.01 & 8.38 & 8.28 & 3.35 & 2.23 & 6.25 \\
\hline \multirow[t]{2}{*}{ Mean } & 7.79 & 6.11 & 4.66 & 3.24 & 1.97 & 4.77 \\
\hline & \multicolumn{3}{|c|}{ S.Em \pm} & \multicolumn{3}{|c|}{ CD (0.01) } \\
\hline Treatment (A) & \multicolumn{3}{|c|}{0.027} & \multicolumn{3}{|c|}{0.074} \\
\hline Genotype (B) & \multicolumn{3}{|c|}{0.068} & \multicolumn{3}{|c|}{0.191} \\
\hline $\mathbf{A} \times \mathbf{B}$ & \multicolumn{3}{|c|}{0.153} & \multicolumn{3}{|c|}{0.426} \\
\hline
\end{tabular}


Table.6 Effect of different concentration of PEG-6000 (control, 4\%, 8\%, 12\% and $16 \%$ on root dry weight $(\mathrm{mg})$ of tomato genotypes

\begin{tabular}{|c|c|c|c|c|c|c|}
\hline \multirow{3}{*}{ Crosses/Hybrids } & \multicolumn{6}{|c|}{ Root dry weight (mg) } \\
\hline & \multicolumn{6}{|c|}{ Treatment } \\
\hline & Control & $4 \%$ & $8 \%$ & $12 \%$ & $16 \%$ & Mean \\
\hline EC-620407 × Arka Saurabh & 10.66 & 7.62 & 6.24 & 3.55 & 3.49 & 6.31 \\
\hline EC-620407 × Arka Abha & 9.93 & 8.18 & 4.47 & 3.83 & 3.04 & 5.89 \\
\hline EC-620407 × Punjab Chhuhara & 5.80 & 5.44 & 5.34 & 3.29 & 3.26 & 4.63 \\
\hline EC-620407 × Pusa Ruby & 5.10 & 4.59 & 4.49 & 3.61 & 3.30 & 4.22 \\
\hline EC-620407 × EC-520078 & 6.26 & 6.06 & 5.42 & 3.51 & 3.08 & 4.87 \\
\hline EC-620407 × EC-620557 & 9.28 & 6.32 & 5.56 & 5.53 & 4.54 & 6.25 \\
\hline EC-620428 × Arka Saurabh & 11.99 & 11.40 & 9.31 & 5.50 & 2.55 & 8.15 \\
\hline EC-620428 × Arka Abha & 8.96 & 8.36 & 6.86 & 2.91 & 2.26 & 5.87 \\
\hline EC-620428 × Punjab Chhuhara & 5.82 & 5.41 & 4.59 & 3.55 & 1.95 & 4.26 \\
\hline EC-620428 × Pusa Ruby & 4.41 & 3.19 & 2.68 & 1.66 & 1.81 & 2.75 \\
\hline EC-620428 × EC-520078 & 3.85 & 3.50 & 3.28 & 3.00 & 2.23 & 3.17 \\
\hline EC-620428 × EC-620557 & 5.22 & 4.30 & 4.28 & 4.18 & 3.59 & 4.31 \\
\hline EC-620360 × Arka Saurabh & 10.65 & 9.62 & 7.64 & 7.52 & 6.11 & 8.31 \\
\hline EC-620360 × Arka Abha & 10.80 & 8.85 & 5.52 & 2.80 & 1.87 & 5.97 \\
\hline EC-620360 × Punjab Chhuhara & 5.46 & 5.42 & 5.28 & 1.94 & 1.18 & 3.85 \\
\hline EC-620360 × Pusa Ruby & 5.05 & 4.63 & 3.81 & 3.22 & 1.46 & 3.63 \\
\hline EC-620360 × EC-520078 & 2.94 & 2.87 & 2.37 & 2.36 & 1.69 & 2.44 \\
\hline EC-620360 × EC-620557 & 4.85 & 2.88 & 2.60 & 2.25 & 1.64 & 2.84 \\
\hline EC-608415 × Arka Saurabh & 5.94 & 4.33 & 4.18 & 3.75 & 2.90 & 4.22 \\
\hline EC-608415 × Arka Abha & 10.22 & 9.83 & 9.55 & 5.37 & 2.94 & 7.58 \\
\hline EC-608415 × Punjab Chhuhara & 6.01 & 7.13 & 4.24 & 2.06 & 1.09 & 4.10 \\
\hline EC-608415 × Pusa Ruby & 4.17 & 4.16 & 4.09 & 3.13 & 1.11 & 3.33 \\
\hline EC-608415 × EC-520078 & 4.10 & 2.25 & 2.13 & 2.10 & 2.06 & 2.53 \\
\hline EC-608415 × EC-620557 & 3.10 & 3.06 & 2.44 & 2.16 & 1.14 & 2.38 \\
\hline EC-619982 × Arka Saurabh & 5.16 & 4.22 & 3.16 & 3.16 & 2.99 & 3.74 \\
\hline EC-619982 × Arka Abha & 9.50 & 4.19 & 4.14 & 3.18 & 3.07 & 4.82 \\
\hline EC-619982 × Punjab Chhuhara & 9.56 & 8.32 & 6.28 & 2.91 & 1.92 & 5.80 \\
\hline EC-619982 × Pusa Ruby & 5.23 & 3.87 & 3.86 & 0.96 & 0.94 & 2.97 \\
\hline EC-619982 × EC-520078 & 3.88 & 3.83 & 3.75 & 2.78 & 0.99 & 3.04 \\
\hline EC-619982 × EC-620557 & 2.10 & 1.97 & 1.93 & 1.91 & 1.84 & 1.95 \\
\hline US-440 & 3.94 & 2.81 & 2.62 & 1.84 & 0.94 & 2.43 \\
\hline Arka Rakshak & 4.90 & 3.94 & 2.94 & 2.82 & 2.80 & 3.48 \\
\hline NS-516 & 8.87 & 8.87 & 8.85 & 3.87 & 3.87 & 6.87 \\
\hline \multirow[t]{2}{*}{ Mean } & 6.47 & 5.49 & 4.75 & 3.21 & 2.41 & 4.47 \\
\hline & \multicolumn{3}{|c|}{ S.Em \pm} & \multicolumn{3}{|c|}{ CD (0.01) } \\
\hline Treatment (A) & \multicolumn{3}{|c|}{0.08} & \multicolumn{3}{|c|}{0.03} \\
\hline Genotype (B) & \multicolumn{3}{|c|}{0.21} & \multicolumn{3}{|c|}{0.07} \\
\hline $\mathbf{A} \times \mathbf{B}$ & \multicolumn{3}{|c|}{0.166} & \multicolumn{3}{|c|}{0.462} \\
\hline
\end{tabular}


Table.7 Effect of different concentration of PEG-6000 (control, 4\%, 8\%, $12 \%$ and $16 \%$ on shoot dry weight $(\mathrm{mg})$ of tomato genotypes

\begin{tabular}{|c|c|c|c|c|c|c|}
\hline \multirow{3}{*}{ Crosses/Hybrids } & \multicolumn{6}{|c|}{ Shoot dry weight (mg) } \\
\hline & \multicolumn{6}{|c|}{ Treatment } \\
\hline & Control & $4 \%$ & $8 \%$ & $12 \%$ & $16 \%$ & Mean \\
\hline EC-620407 × Arka Saurabh & 79.78 & 70.75 & 49.56 & 29.74 & 28.14 & 51.59 \\
\hline EC-620407 × Arka Abha & 76.77 & 58.25 & 45.04 & 33.80 & 24.68 & 47.71 \\
\hline EC-620407 × Punjab Chhuhara & 85.25 & 77.99 & 58.16 & 33.07 & 24.68 & 55.83 \\
\hline EC-620407 × Pusa Ruby & 91.71 & 62.50 & 54.50 & 42.45 & 41.25 & 58.48 \\
\hline EC-620407 × EC-520078 & 83.07 & 51.81 & 46.56 & 46.56 & 33.93 & 52.37 \\
\hline EC-620407 × EC-620557 & 74.08 & 54.73 & 46.75 & 40.05 & 31.51 & 49.42 \\
\hline EC-620428 × Arka Saurabh & 88.07 & 87.78 & 85.78 & 43.52 & 33.04 & 67.64 \\
\hline EC-620428 × Arka Abha & 82.07 & 71.04 & 65.94 & 26.99 & 21.25 & 53.46 \\
\hline EC-620428 $\times$ Punjab Chhuhara & 74.62 & 57.28 & 53.94 & 35.49 & 30.79 & 50.42 \\
\hline EC-620428 × Pusa Ruby & 82.42 & 43.57 & 41.12 & 30.66 & 23.41 & 44.23 \\
\hline EC-620428 × EC-520078 & 67.55 & 50.73 & 47.48 & 33.81 & 30.44 & 46.00 \\
\hline EC-620428 × EC-620557 & 80.31 & 63.96 & 48.88 & 43.44 & 28.56 & 53.01 \\
\hline EC-620360 × Arka Saurabh & 86.47 & 85.78 & 50.36 & 50.35 & 35.95 & 61.78 \\
\hline EC-620360 × Arka Abha & 86.46 & 62.26 & 32.55 & 24.25 & 19.46 & 44.99 \\
\hline EC-620360 × Punjab Chhuhara & 72.76 & 70.23 & 55.50 & 28.94 & 22.83 & 50.05 \\
\hline EC-620360 × Pusa Ruby & 70.52 & 68.54 & 51.92 & 34.63 & 22.19 & 49.56 \\
\hline EC-620360 × EC-520078 & 82.38 & 47.37 & 42.33 & 34.99 & 24.52 & 46.32 \\
\hline EC-620360 × EC-620557 & 43.18 & 43.00 & 40.47 & 31.59 & 28.25 & 37.30 \\
\hline EC-608415 × Arka Saurabh & 70.25 & 68.14 & 44.24 & 34.49 & 29.42 & 49.31 \\
\hline EC-608415 × Arka Abha & 86.05 & 86.00 & 84.12 & 49.54 & 28.59 & 66.86 \\
\hline EC-608415 × Punjab Chhuhara & 70.42 & 66.06 & 61.15 & 18.25 & 16.59 & 46.49 \\
\hline EC-608415 × Pusa Ruby & 52.74 & 47.91 & 29.55 & 27.17 & 19.86 & 35.44 \\
\hline EC-608415 × EC-520078 & 69.13 & 66.55 & 32.59 & 22.63 & 22.38 & 42.66 \\
\hline EC-608415 × EC-620557 & 82.30 & 41.66 & 38.32 & 36.18 & 27.17 & 45.13 \\
\hline EC-619982 × Arka Saurabh & 66.80 & 65.68 & 37.31 & 35.41 & 26.41 & 46.32 \\
\hline EC-619982 × Arka Abha & 83.52 & 48.75 & 42.35 & 31.64 & 26.78 & 46.61 \\
\hline EC-619982 × Punjab Chhuhara & 84.82 & 84.36 & 26.65 & 19.20 & 15.17 & 46.04 \\
\hline EC-619982 × Pusa Ruby & 64.35 & 57.93 & 56.47 & 25.36 & 15.38 & 43.90 \\
\hline EC-619982 × EC-520078 & 50.13 & 46.36 & 26.53 & 25.28 & 16.39 & 32.94 \\
\hline EC-619982 × EC-620557 & 66.52 & 60.61 & 31.07 & 19.70 & 18.39 & 39.26 \\
\hline US-440 & 80.38 & 40.04 & 31.69 & 31.58 & 24.45 & 41.63 \\
\hline Arka Rakshak & 62.06 & 60.75 & 33.58 & 33.04 & 25.59 & 43.00 \\
\hline NS-516 & 83.17 & 81.42 & 80.38 & 30.45 & 23.02 & 59.69 \\
\hline \multirow[t]{2}{*}{ Mean } & 75.15 & 62.11 & 47.66 & 32.85 & 25.46 & 48.75 \\
\hline & \multicolumn{3}{|c|}{ S.Em \pm} & \multicolumn{3}{|c|}{ CD $(0.01)$} \\
\hline Treatment (A) & \multicolumn{3}{|c|}{0.197} & \multicolumn{3}{|c|}{0.549} \\
\hline Genotype (B) & \multicolumn{3}{|c|}{0.506} & \multicolumn{3}{|c|}{1.41} \\
\hline $\mathbf{A} \times \mathbf{B}$ & \multicolumn{3}{|c|}{1.133} & \multicolumn{3}{|c|}{3.152} \\
\hline
\end{tabular}




\section{Shoot Length $(\mathrm{cm})$}

The results revealed that there is a significant difference in shoot length between the genotypes and different PEG-6000 concentrations (Table 5). Irrespective of the PEG-6000 concentrations, EC-620360 × Arka Saurabh recorded significantly maximum shoot length $(6.85 \mathrm{~cm})$ compared to all other genotypes. The shoot length was lowest $(2.66$ $\mathrm{cm})$ in the genotype EC-619982 $\times$ EC-620557. The interaction effect between the genotype and PEG-6000 concentrations was found significant. In $4 \%$ concentration of PEG-6000, maximum and minimum shoot length was recorded in EC-620360 $\times$ Arka Saurabh (9.69 $\mathrm{cm})$ and EC-619982 × EC-620557 $(3.11 \mathrm{~cm})$ genotype respectively. While at $8 \%$ concentration of PEG-6000, genotype EC$608415 \times$ Arka Abha $(8.18 \mathrm{~cm})$ and Arka Rakshak (2.40) exhibited maximum and minimum shoot length respectively. In case of $12 \%$ concentration of PEG-6000, genotypes EC-620360 × Arka Saurabh (5.28) and EC$608415 \times$ Pusa Ruby $(0.70 \mathrm{~cm})$ had responded as maximum and minimum shoot length respectively. In case of $16 \%$ concentration of PEG-6000, genotypes EC-620407 × Pusa Ruby (4.28) and EC-619982 × EC-620557 $(0.27 \mathrm{~cm})$ had responded as maximum and minimum shoot length respectively.

Genotypes with indeterminate growth habit showed more reduction in shoot length as compared to determinate type. It indicates that determinate tomato can be well suited to drought areas than indeterminate growth habit. Turner (1979) has recommended indeterminate growth habit for drought tolerance. But the results of present experiment advocate determinate growth habit for drought resistance. Present experimental results were similar to earlier studies of Govindaraj (2010) and Kulkarni and Desphpande (2007). It is generally accepted that the roots suffer first from exposure to stresses, followed by their associated plant parts (Misra and Dwivedi, 2004 and Abida et al., 2013).

\section{Root dry weight (mg)}

Highest root weight was recorded by EC$620360 \times$ Arka Saurabh (8.31) (Table 6). Regardless of the PEG-6000 concentrations, the root dry weight was lowest (2.43) in the genotype US-440. The interaction effect between the genotype and PEG-6000 concentrations was found significant. In $4 \%$ concentration of PEG-6000, maximum and minimum root dry weight was recorded in EC$620428 \times$ Arka Saurabh $(11.40 \mathrm{mg})$ and EC619982 x EC-620557 (1.97 mg) genotype respectively. While at $8 \%$ concentration of PEG-6000, genotype EC-620428 $\times$ Arka Saurabh (9.31 mg) and EC-619982 x EC620557 (1.93 mg) exhibited maximum and minimum root dry weight respectively. In case of $12 \%$ concentration of PEG-6000, genotypes EC-620360 × Arka Saurabh (7.52 mg) and EC$619982 \times$ Pusa Ruby $(0.96 \mathrm{mg})$ had responded as maximum and minimum root dry weight respectively. With $16 \%$ concentration of PEG6000, genotypes EC-620360 × Arka Saurabh $(6.11 \mathrm{mg})$ and EC-619982 × Pusa Ruby (0.94 $\mathrm{cm}$ ) had responded as maximum and minimum root dry weight respectively

Growth parameters like dry weight is known to have a profound effect on water limited conditions. In the present study a reduction in root dry weight was recorded in stressed conditions in all the genotypes. Present investigation is in confirmation with Ali et al., 1999, Bhatt and Srinivas (1987), Umesh Chandra and Gupta (1994), Deshpande and Kulakarni (2005), Ragab et al., (2007), Wani et al., (2010) and Tyagi et al., (1995). Root dry weight depends on the germination percent and root length, low water uptake and restricted metabolic activities were given to decrease in the root dry weight. Most of the tolerant genotypes accumulated more dry matter under control that too increased under stress however moderately tolerant or susceptible genotypes recorded comparatively less increase or even decrease in dry weight of roots. 


\section{Shoot dry weight (mg)}

Irrespective of the PEG-6000 concentrations, EC-620428 $\times$ Arka Saurabh recorded significantly maximum shoot dry weight (67.64 $\mathrm{mg}$ ) compared to all other genotypes (Table 7). Significant differences were observed for shoot dry weight between the genotypes and different PEG-6000 concentrations.

The shoot dry weight was lowest $(32.94 \mathrm{mg})$ in the genotype EC-619982 × EC-520078. The interaction effect between the genotype and PEG-6000 concentrations was found significant. In $4 \%$ concentration of PEG-6000, maximum and minimum shoot dry weight was recorded in EC-620428 × Arka Saurabh (87.78 $\mathrm{mg})$ and US-440 (40.04 mg) genotype respectively. While at $8 \%$ concentration of PEG-6000, genotype in EC-620428 $\times$ Arka Saurabh $(85.78 \mathrm{mg})$ and EC-619982 $\times$ Punjab Chhuhara $(26.65 \mathrm{mg})$ exhibited maximum and minimum shoot dry weight respectively. In case of $12 \%$ concentration of PEG-6000, genotypes EC-620360 × Arka Saurabh (50.35 mg) and EC-608415 $\times$ Punjab Chhuhara $(18.25 \mathrm{mg})$ had responded with maximum and minimum shoot dry weight respectively. In case of $16 \%$ concentration of PEG-6000, genotypes EC$620407 \times$ Pusa Ruby (41.25 mg) and EC$619982 \times$ EC-520078 $(16.39 \mathrm{mg})$ had responded with maximum and minimum shoot dry weight respectively. These results are in accordance with Kulkarni and Deshpande (2006).

Among all crosses, EC-620428 × Arka Saurabh, EC-620360 $\times$ Arka Saurabh, EC-620427 $\times$ EC620557 responded superior performance compared to others. Difference in sensitivity and response of plants to PEG at cultivar level largely depends upon genetic constitution of the plants (Garsia-Reina et al., 1988). According to Imanparast and Hassanpanah (2009) genotypes that had good root length had excess germination percentage too and seeds had good root growth. These results for seedling level stress tolerance were correlated with the findings of Fischer and Maurer, (1978). Hence, genotypes with higher germination percentage, root length and dry weights should be selected while screening for drought tolerance. These genotypes may be used as positive/tolerant controls in future studies. However, the internal physiological investigation is needed for assessing their variable response.

\section{References}

Aazami, M.A., Torabi, M. and Jalili E. 2010. In vitro response of promising tomato genotypes for tolerance to osmotic stress. African Journal of Biotechnology. 9(26): 4014-4017.

Abdel-Raheem, A. T., Kasem, Z. A., Omar, F. D. and Samera A.M. 2007. Evaluation of R1 tomato somaclone plants selected under poly ethylene glycol (PEG) treatments. African Crop Science Conference Proceedings. 8: 2017-2025.

Abida, R. Ashraf, M. and Ghafoor A. 2013. Genetic divergence for seedling traits in tomato (Solanum lycopersicum). International of Journal Agricultural Biology. 15: 451-457.

Alejandra Nieto-Garibay, Bernardo MurilloAmador, Enrique Troyo-Dieguez, Jose L. Garcia-Hernandez and Francisco H. RuizEspinoza 2010. Water stress in two capsicum species with different domestication grade. Tropical and Subtropical Agroecosystems. 12: 353 - 360.

Ali, M., Jensen, C. R., Mogensen, V. O., Andersen, M. N. and Henson I. E. 1999. Root signalling and osmotic adjustment during intermittent soil drying sustain grain yield of field grown wheat. Field Crop Research. 62: 35-52.

Aslam, Iftikhar A. Khan, Muhammad Saleem and Zulfiqar Ali 2006. Assessment of water stress tolerance in different maize accessions at germination and early growth stage. Pakistan Journal of Botany. 38(5): 1571-1579.

Babu, L., Muthukrishnan, C. R. and Irulappan I. 1985. Studies on drought tolerance in tomato. South Indian Horticulture. 33: 377380.

Bhatt, R. M. and Srinivas Rao N. K. 1987. Seed 
germination and seedling growth responses of tomato cultivars to impose water stress. Journal of Horticultural Science. 62: 221225.

Bressan, R.A., P.M. Hasegawa and Handa A.K. 2003. Resistance of cultured higher plant cells to polyethylene glycol-induced water stress. Plant Science Letters. 21: 23-30.

Deshpande U. D. and Manoj Kulkarni 2005. Root anatomical and morphological basis for drought resistance in tomato. ICPSBC2005. Abstract Number-BT·8/31 (b).

Fischer, R. A. and Maurer R. 1978. Drought resistance in spring wheat cultivars. I. Grain yields responses. Australian Journal of Agricultural Research. 29: 897- 912.

Garsia-Reina, G. Moreno, V. and Luque A. 1988. Selection for $\mathrm{NaCl}$ tolerance in cell culture of three canary island tomato land races. Recovery of tolerant plantlets from $\mathrm{NaCl}$ tolerant cell strains. Journal of Plant Physiology. 133: 1-6.

Gopal, J. and Iwama K. 2007. In vitro screening of potato against water stress mediated through sorbitol and polyethylene glycol. Plant Cell Reports. 26: 693-700.

Govindaraj, M., P. Shanmugasundaram, P. Sumathi and Muthiah A.R. 2010. Simple, rapid and cost effective screening method for drought resistant breeding in pearl millet. Electronic Journal of Plant Breeding. 1: 590- 599.

Hanson, A.D. Rathinasabapathi, B. Rivoal, J. Burnet, M. Dillon, M.O. and Gage D.A. 1994. Osmoprotective compounds in the Plubaginaceae: A natural experiment in metabolic engineering of stress tolerance. Proceedings of National Academy of Sciences. 91: 306-310.

Imanparast, L. and Hassanpanah D. 2009. Response of Onobrychis genotypes to PEG 10000 induced osmotic stress. Biotechnology. 8(3): 365-369.

Jurekova, Z., K. Nemeth-Molnar and Paganova V. 2011. Physiological responses of six tomato (Lycopersicon esculentum Mill.) cultivars to water stress. Journal of Horticulture and Forestry. 3: 294-300.

Kalefetogllu, T. Macar, T. Turan, O. and Ekmekci Y. 2009. Effect of water deficit induced by PEG and $\mathrm{NaCl}$ on Chickpea (Cicer arieitinum L.) cultivar and lines at early seedling stage. Gazi University Journal of Science. 22(1): 5-14.

Khodarahmpour, Z. 2011. Effect of drought stress induced by polyethylene glycol (PEG) on germination indices in corn (Zea mays L.) hybrids. African Journal of Biotechnology. 10: 18222-18227.

Kim, Y. J. Shanmugasundaram, S. Yun, S. J. Park, H. K. and Park M.S. 2001. A simple method of seedling screening for drought tolerance in soybean. Korean Journal of Crop Science. 46: 284-288.

Kulkarni, M. and Deshpande U. 2006. Anatomical breeding for altered leaf parameters in Tomato genotypes imparting drought resistance using Leaf Strength Index. Asian Journal of Plant Sciences. 5(3): 414-420.

Kulkarni, M. and Deshpande U. 2007. In vitro screening of tomato genotypes for drought resistance using polyethylene glycol. African Journal of Biotechnology. 6(6): 691-696.

Larher, F., L.Leport, M. Petrivalskyand and Chappart M. 1993. Effectors for the osmoinduced proline response in higher plants. Plant Physiology and Biochemistry. 31: 911-922.

Leishman M.R, and Westoby M. 1994. The role of seed mass in seedling establishment in dry soil conditions - experimental evidence from semi-arid species. Journal of Ecology. 82: $249-258$.

Munns, R. and Weir R. 1981. Contribution of sugars to osmotic adjustment in elongating and expanding zones of wheat leaves during moderate water deficits at two light levels. Australian Journal of Plant Physiology. 8: 93- 105.

Mishra Neelum and Dwivedi 2004. Genotypic difference in salinity tolerance of green gram cultivars. Plant Science. 166(5): $1135-1142$.

Morgan, J. M. 1992. Osmotic components and properties associated with genotypic differences in osmoregulation in wheat. Australian Journal of Plant Physiology. 19: 67-76.

Narayan, D. 1991. Root growth and productivity of wheat cultivars under different soil moisture conditions. International Journal 
of Ecology and Environmental Science. 17: 19-26.

Nepomuceno, A. L. Oostrerhuis, D. M. and Stewart J. M. 1998. Physiological responses of cotton leaves and roots to water deficit induced by polyethylene glycol. Environment and Experimental Botany. 40: 29-41.

Panchalingam, S. 1983. Studies on drought tolerance in brianjal (Solanum melongena L.) M.Sc (Hort.) Thesis, TNAU, Coimbatore, India.

Pena, R.D.L. and Hughes J. 2007. Improving vegetable productivity in a variable and changing climate. SAT eJournal, 4: 1-22.

Ragab, A. R. Abdel-Raheem, A. T. Kasem, Z. A. Omar, F. D. and Samera A. M. 2007. Evaluation of R1 tomato somaclone plants selected under poly ethylene glycol (PEG) treatments. African Crop Science Conference Proceedings. 8:2017-2025.

Rao and Bhatt 1991. Tolerance to Water Stress in Tomato Cultivars. Photosynthetica. 38(3): 465-467.

Ravi ranjan Kumar, Karjol, K. and Naik G. R. 2011. Variation of sensitivity to drought stress in Pigeon pea (Cajanus cajan (L) cultivars during seed germination and early seedling growth. World Journal of Science and Technology. 1(1): 11-18.

Rizvi Abida, Muhammad Ashraf and Abdul Ghafoor 2013. Genetic divergence for seedling traits in tomato (Solanum lycopersicum). International Journal of Agriculture and Biology. 15: 451-457.

Sakthivelu, G., M.K. A. Devi, P. Giridhar, T. Rajasekaran, G.A. Ravishankar, T. Nedev and Kosturkova G. 2008. Drought induced alterations in growth, osmotic potential and in vitro regeneration of soybean cultivars. Applied Plant Physiolology. 34: 103-112.

Steel, R.G.D., J.H. Torrie and Dickey D.A. 1997. Principles and Procedures of Statistics: A Biometrical Approach. 3rd Ed. McGraw Hill Book Co. Inc. New York, USA.

Tyagi, A., Santha, I. M. and Mehta S. L. 1995. Molecular response to water stress in Lathyrus sativus. Journal of Plant Biochemistry and Biotechnology. 4: 47-49.

Umesh chandra. And Gupta P. N. 1994. Evaluation of tomato germpasm adaptable to abiotic stress conditions of Northern India. Indian Journal of Plant Genetic Resources. 7(2): 165-172.

Uniyal, R. C. and Nautiyal A. R. 1998. Seed germination and seedling extension growth in Ougeinia dalbergioides benth. Under water and salinity stress. New Forests. 16: 265-272.

Unyayar S., Y. Kelesand and Cekic F.O. 2005. The antioxidative response of two tomato species with different drought tolerances as a result of drought and cadmium stress combinations. Plant Soil Environment. 51: 57-64.

Wani, S. H. Sofi, P. A. Gosal, S. S. and Singh N. B. 2010. In vitro screening of rice (Oryza sativa L) callus for drought tolerance. International Journal of the Faculty of Agriculture and Biology. 5(2): 108-115.

Zhu, J. Kang, H. Tan, H. and Xu M. 2006. Effects of drought stresses induced by polyethylene glycol on germination of Pinus sylvestris var. mongolica seeds from natural and plantation forests on sandy land. Journal of Forest Research. 11: 319-328.

\section{How to cite this article:}

Arun Kumar, P., N.N. Reddy and Jyothi Lakshmi, N. 2017. PEG Induced Screening for Drought Tolerance in Tomato Genotypes. Int.J.Curr.Microbiol.App.Sci. 6(7): 168-181. doi: https://doi.org/10.20546/ijcmas.2017.607.020 